\title{
Räumliches Planen in Wissenschaft und Praxis - von „Mind the Gap“ zu ,Finding Gaps“
}

\author{
Meike Levin-Keitel ${ }^{1}$ Martin Sondermann²
}

Online publiziert: 2. Januar 2017

(C) Springer-Verlag Berlin Heidelberg 2017

Räumliche Planung ist ein weites Handlungsfeld und umfasst sowohl die Planungspraxis im Sinne planerischer Interventionen in räumliche Entwicklungen als auch die Planungswissenschaft als korrespondierende wissenschaftliche Disziplin. Wissenschaft und Praxis unterscheiden sich dabei schon allein aufgrund ihrer unterschiedlichen Denkund Handlungsweisen, ihrer Kontexte, Anforderungen und Möglichkeiten. Wir wollen in diesem Schwerpunktheft den Fragen nachgehen, in welchem Verhältnis Planungswissenschaft und -praxis heute zueinander stehen, inwiefern sie füreinander von Bedeutung sind und welche Rolle Theorien dabei spielen.

Ausgehend von der klassischen Forderung, sich mit der Theorie-Praxis-Lücke zu beschäftigen - „Mind the Gap“ -, zeigen wir in diesem Heft eine andere Deutungsweise auf, welche sich nur bedingt anhand der Dichotomie Theorie und Praxis erklären lässt, sondern vielmehr von einer vielfältigen Dreiecksbeziehung von Wissenschaft, Theorie und Praxis ausgeht, und sich auf die Suche nach ,blinden Flecken“ begibt - „Finding Gaps“. Zur Einführung in dieses Schwerpunktheft werden hierzu erste grundsätzliche Überlegungen überblicksartig dargestellt. Sie basieren auf den Diskussionen des Arbeitskreises „Mind the Gap“ der Aka-

Dr. Meike Levin-Keitel

levin@umwelt.uni-hannover.de

Martin Sondermann

sondermann@arl-net.de

1 Institut für Umweltplanung, Leibniz Universität Hannover, Herrenhäuser Straße 2, 30419 Hannover, Deutschland

2 Akademie für Raumforschung und Landesplanung, Leibniz-Forum für Raumwissenschaften, Hohenzollernstraße 11, 30161 Hannover, Deutschland demie für Raumforschung und Landesplanung (ARL) sowie einer internen Umfrage unter den Mitgliedern ${ }^{1}$.

\section{1 Über räumliche Planung als Praxis}

Das Verständnis davon, was räumliche Planung ist bzw. umfasst, kann nicht allgemeingültig beschrieben werden: Anstatt von einer räumlichen Planung im Singular zu sprechen, sehen wir eine Vielfalt an räumlichen Planungen, welche allgemein-integrierend (Stadt-, Regional- und Landesplanung) oder fachspezifisch (wie z. B. Landschafts- oder Verkehrsplanung) arbeiten und damit unterschiedliche,Subkulturen ' innerhalb der Disziplin bilden. Diese Subkulturen zeichnen sich unter anderem dadurch aus, dass auf verschiedenen räumlichen Maßstabsebenen und mit unterschiedlichen Anteilen politisch-administrativer, ingenieurtechnischer und sozial-kommunikativer Logiken gedacht und gehandelt wird.

Im Wesentlichen umfasst die Planungspraxis alle konkreten Handlungen, welche sich über Aushandlungs- und Entscheidungsprozesse auf räumliche Entwicklungen auswirken. Diese Prozesse werden von unterschiedlichsten Akteuren vollzogen, wozu klassischerweise Planerinnen und Planer in der Verwaltung gehören sowie private Planungsbüros, Akteure aus der Zivilgesellschaft und Träger öffentlicher Belange. Die Planungsverwaltungen nehmen dabei nach wie vor eine privilegierte Position ein, denn sie verfügen als Teil des politisch-administrativen Systems über eine

\footnotetext{
${ }^{1}$ Mitglieder des Arbeitskreises sind Meike Levin-Keitel und Martin Sondermann (Leitung), Christian Diller, Evelyn Gustedt, Meike Hellmich, Yvonne Knapstein, Christian Wilhelm Lamker, Linda Lange, Annick Leick, Bettina Lelong, Marlene Lerch, Ulrike Mackrodt, Christian Peer und Thomas Thaler.
} 
hoheitliche Machtposition sowie über eine gewisse Deutungshoheit in planerischen Aushandlungsprozessen.

In der Planungspraxis werden bestimmte Instrumente, Methoden und Verfahren angewandt, um zukünftige räumliche Entwicklungen zu steuern bzw. zu beeinflussen. Neben klassischen Verfahren der Bauleitplanung werden auch Entwicklungsprogramme durchgeführt, Nutzungskonflikte moderiert, Interessen abgewogen und kommunikative Prozesse gestaltet. Dabei sind die Gegenstände, Ziele, Methoden, Verfahrensweisen und beteiligten Akteure räumlichen Planens immer für den Einzelfall genau zu definieren und es ist die Frage zu klären, welche Rolle die Planung in der räumlichen Entwicklung übernehmen kann und soll. Obgleich einer prinzipiellen Offenheit gegenüber der Frage, was Planung umfasst, vertreten wir die Auffassung, dass sich Planungspraktiken stets am Gemeinwohl orientieren und ihre zentralen Arbeitsprinzipien im Abwägen und Koordinieren von Belangen und Interessen bestehen, womit ihnen eine zentrale Funktion in einer demokratischen Raumentwicklung zukommt.

\section{Theorien der Praxis - ,Mind the Gap“}

Die theoretischen Abbilder der Planungspraxis werden in der Fachwelt schon seit geraumer Zeit kritisch diskutiert und es wird aufgefordert, sich explizit mit dem TheoriePraxis-Verhältnis zu beschäftigen - „Mind the Gap“ (Allmendinger/Tewdwr-Jones 1997). So erscheinen Planungstheorien inadäquat, wenn sie mit praktischen Erfahrungen nicht vereinbar sind bzw. praktisches Handeln nicht unterstützen (Innes de Neufville 1983: 35). Um die Theorie-Praxis-Lücke zu überbrücken - „Bridging the Gap“ -, plädiert Innes de Neufville (1983: 35) für neue theoretische Bilder von empirisch beobachtbaren Planungspraktiken, welche die realen Bedingungen und Schwierigkeiten des Planens berücksichtigen. Bis heute wird in der wissenschaftlichen Diskussion allerdings immer wieder auf das Bild einer Lücke (,the Gap“) zurückgegriffen (u. a. Alexander 1997; Gualini 2010; Pissourios 2013; Lord 2014).

Gegenständlich befassen sich die Autorinnen und Autoren dieses Schwerpunktheftes erstens mit dem Theorie-Praxis-Verhältnis hinsichtlich der komplexen Kontexte, spezifischen Situationen und realen Bedingungen, in denen Planungspraktiken vollzogen werden. Zweitens geht es um die Übersetzung theoretischen Wissens in praktisches Handeln (vgl. Allmendinger/Tewdwr-Jones 1997) sowie drittens um die zugrunde liegende Produktion theoretischen Wissens (vgl. Van de Ven/Johnson 2006: 802 f.). So wird unter anderem die Art und Weise kritisiert, wie die Praxis betrachtet und interpretiert wird, und auch ein nichttheoretisches Verständnis von Planung diskutiert (vgl. Lord 2014).
All diese Beiträge zur Planungstheorie sind allerdings im Feld der Planungswissenschaft zu verorten: Dort kritisieren Wissenschaftlerinnen und Wissenschaftler diejenigen Theorien, die in der Planungswissenschaft produziert wurden, weshalb es sich bei dem Theorie-Praxis-Verhältnis streng genommen um ein Wissenschaft-Praxis-Verhältnis handelt. Hinzu kommt, dass jedes praktische Handeln mit gedanklichen Konstruktionen, also Theorien, korrespondiert - sei es explizit oder implizit, auf wissenschaftlichem oder subjektivem Wissen basierend.

\section{Theorien in Planungswissenschaft und -praxis - ,Finding Gaps“6}

Bei Planungstheorien handelt es sich aus unserer Sicht um abstrahierte, gedankliche Konstrukte, welche räumliches Planen in seinen Inhalten und Verfahrensweisen (substanziell/prozedural) abbilden. Diese Theorien können sowohl nach wissenschaftlichen Regeln konstruiert werden als auch auf Erfahrungswissen basieren, also sowohl in der Planungswissenschaft als auch in der Planungspraxis generiert werden (vgl. Abbildung 1). Alle Theorien entstehen unserer Ansicht nach nicht in grundsätzlich verschiedenen Handlungsfeldern, sondern in einem Kontinuum aus wissenschaftlichen und praktischen Beobachtungen, Deutungen und Abstraktionen planerischer Realitäten. Planungstheorien werden in diesem Sinne aus den beiden der Theorie nachgelagerten Handlungsfeldern der Wissenschaft und Praxis gespeist, zu veränderlichen Anteilen.

Planungstheorien, welche primär in der Wissenschaft generiert werden, haben häufig einen Modellcharakter, sollen also die komplexen Wirklichkeiten anschaulich reduzieren, bestimmte Ausschnitte der räumlichen Planung erklären oder als Analysemodelle der empirischen Erforschung planerischer Praktiken dienen. Hierzu werden Ansätze aus anderen Disziplinen auf die räumliche Planung übertragen, womit sich das Wissensfeld stets neu konstituiert und verschiedene Denkweisen (z. B. aus den Sozial-, Kultur- und Wirtschaftswissenschaften) in die Planung integriert werden. Dabei stehen die vorwiegend geisteswissenschaftlich fundierten, wissenschaftlich generierten Planungstheorien in einem gewissen Widerspruch zu einer handlungsorientierten, ingenieurwissenschaftlichen Denkweise, welche die Praxis nach wie vor prägt: So können (geisteswissenschaftlich-) theoretische Ansätze nicht als (ingenieurtechnisch-) praktische Anleitungen fungieren (vgl. Alexander 1997: 5; Alexander 2010: $103 \mathrm{f}$.).

Bei Theorien handelt es sich immer um selektive, modellhafte Vorstellungen einer viel komplexeren Realität. Dies kann zum Beispiel an kommunikativen Planungstheorien nachvollzogen werden, welche die Vorteile kommunikativer Aushandlung und kooperativer Zusammenarbeit ge- 


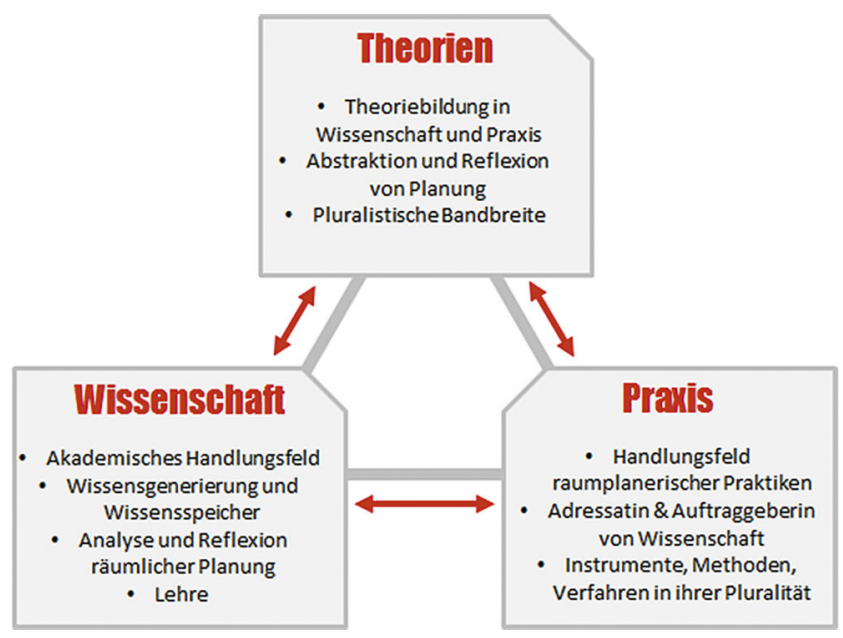

Abbildung 1 Verhältnisse der Dreiecksbeziehung WissenschaftTheorien-Praxis

wissermaßen bewerben und beispielsweise auf die „Macht des besseren Arguments“ setzen (vgl. u. a. Healey 1996: 219). Dieses Ideal steht jedoch häufig im Widerspruch zu Planungspraktiken, wo viele Entscheidungen jenseits ,guter Argumente" erfolgen (vgl. Lord 2014: 30). Neben den zahlreichen wissenschaftlichen Theorien zum kommunikativen Planen gibt es unzählige in der Praxis generierte Theorien darüber, wie - basierend auf den Erfahrungen im praktischen Vollzug - kommunikative Planung funktioniert, funktionieren sollte und wo (instrumentelle, methodische, verfahrenstechnische etc.) Möglichkeiten und Grenzen bestehen. Von welchen Bedingungen und Faktoren das reale Planen abhängt, wird dabei nie lückenlos von der Forschung erfasst. So wurden Aspekte wie die Verteilung von Macht (vgl. Reuter 2000) oder kulturelle Prägungen (vgl. Levin-Keitel/Sondermann 2015) in der Wissenschaft lange nicht hinreichend berücksichtigt, auch wenn sie im Alltag von Planerinnen und Planern von Bedeutung sind.

Die Planungswissenschaft ist ein Tätigkeitsfeld, in dem sich Wissenschaftlerinnen und Wissenschaftler gegenständlich mit räumlicher Planung befassen, diese empirisch erforschen und als Lehrende zukünftige Planerinnen und Planer ausbilden. Die Besonderheit der Planungswissenschaften besteht darin, dass es ein direkt korrespondierendes Handlungsfeld gibt. Daraus ergibt sich ein gewisser Anspruch an die Anwendbarkeit planungswissenschaftlicher Forschung und Lehre, welche für andere Disziplinen (wie der Geographie) nicht bestehen, da diese keinen unmittelbaren Berufs- und Handlungsbereich ansprechen. Der Beitrag der Planungswissenschaft für die Planungspraxis besteht beispielsweise darin, dass sie

- die theoretischen Grundlagen für das planerische Handeln liefert, also Wissen darüber, was und wie geplant wird (vgl. auch Allmendinger 2009: 11),
- planerisches Wissen aufbereitet, schriftlich fixiert und damit eine Grundlage für die universitäre Ausbildung und den fachlichen Austausch schafft,

- strukturelle und prozessuale Stärken und Schwächen erkennt und Anpassungsmöglichkeiten aufzeigt und

- ein Angebot von zahlreichen Planungstheorien schafft, welche Praktikerinnen und Praktiker je nach Aufgabe und Situation zur Unterstützung und Rechtfertigung des eigenen Handelns nutzen können (vgl. auch Allmendinger/Tewdwr-Jones 1997: 803).

Die Planungspraxis ist ihrerseits von essenzieller Bedeutung für die Planungswissenschaft, als dass sie

- deren Forschungsbereich definiert und den empirischen Pool für die wissenschaftliche Beschäftigung bildet,

- sich stets verändert und dementsprechend einen Forschungsbedarf generiert, ,neue Praktiken“ in ihren jeweiligen Kontexten wissenschaftlich zu untersuchen bzw. neue Theorien der Praxis zu entwickeln (vgl. Alexander 1997) und

- Adressatin und Auftraggeberin planungswissenschaftlicher Forschung ist.

Die Bedeutung von Planungstheorien in beiden Feldern ist dabei ausgesprochen vielschichtig: Zum einen stellen sie durch ihre gegenständliche Befassung mit räumlicher Planung den zentralen, gedanklichen Bezugsrahmen her. Auch wenn keine Theorie praktisches Handeln lückenlos erklären oder anleiten kann, so vermag die Vielzahl an Theorien doch die Disziplin in ihrer Heterogenität annäherungsweise zu repräsentieren. Zum anderen dienen Theorien dazu, die Disziplin der räumlichen Planung zu festigen und ihre Rationalität, Legitimität und Effektivität zu erhöhen. Von zentraler Bedeutung erscheint uns die Weiterentwicklung der Disziplin durch Theorien als Medium zur Selbstreflexion und kritischen Hinterfragung des (eigenen) planerischen Tuns sowie als Inspirationsquelle für neue Instrumente, Methoden und Verfahrensweisen (vgl. auch Alexander 2010). Theorien beschreiben demnach abstrahiertes und generalisiertes Planungswissen, generiert in beiden Handlungsfeldern, den Wissenschaften wie auch der Planungspraxis.

So basiert der Wissenstransfer zwischen Wissenschaft und Praxis maßgeblich auf Theorien, welche unter anderem durch universitäre Lehre, wissenschaftliche Veröffentlichungen und kommunikativen Austausch zwischen beiden Feldern diffundieren. Auf der Suche nach möglichen Diskrepanzen, Problemen und Unstimmigkeiten zwischen wissenschaftlichen und praktischen Sichtweisen auf das räumliche Planet deuten sich hier eine Vielzahl von Lücken im Sinne ,blinder Flecken ' an, da sich sowohl der wissenschaftliche Diskurs als auch die gelebte Praxis fortwährend wandeln und damit - je nach Perspektive und Betrachtungsgegenstand - immer wieder neue Notwendigkeiten und Mög- 
lichkeiten der Wissensproduktion in beiden Feldern ergeben. In diesem Sinne gibt es keine Lücke zwischen Theorie und Praxis räumlichen Planens, gleichwohl aber jede Menge ,blinde Flecken' in der Disziplin, welche es zu finden gilt - im Sinne von „Finding Gaps“.

\section{Beiträge zu ,Finding Gaps“ in diesem Schwerpunktheft}

Auf der Suche nach diesen ,blinden Flecken ' wurden für dieses Schwerpunktheft Beiträge ausgesucht, die die vielseitigen Verhältnisse von Wissenschaft, Praxis und Theorien räumlichen Planens auf unterschiedliche Weise analysieren und diskutieren. Wir beginnen die Suche im alltagspraktischen Handeln von Regionalplanerinnen und Regionalplanern. Diese wurden für den Beitrag von Meike Hellmich, Christian Wilhelm Lamker und Linda Lange zu ihren Sichtweisen auf und Verhältnissen zu Wissenschaft und Theorien befragt. Dabei wird unter anderem aufgezeigt, dass wissenschaftliche Erkenntnisse und Transdisziplinarität von Praktikerinnen und Praktikern als bedeutsam für ihr Handeln erachtet werden. Zugleich wird aber auch deutlich, dass die Vielfalt planerischer Praktiken nicht von Theorien abgedeckt wird, sprich ,blinde Flecken` bleiben.

Ein konkretes Beispiel für eine Wahrnehmungslücke seitens der Planungswissenschaft ist die ,ressortfremde Planungspraxis“, mit der sich Ulrike Mackrodt und Marlene Lerch in ihrem Beitrag beschäftigen. Ausgehend von einer Betrachtung von Projektförderungen erörtern die beiden Autorinnen am Beispiel Berlins, wie sich Planung auch jenseits institutioneller Grenzen vollzieht, und plädieren für eine offene wissenschaftliche Beschäftigung mit dem weiten empirischen Feld räumlichen Planens.

Dieses Feld wird in zwei weiteren Beiträgen auf die kommunikativ-kooperative Planung eingegrenzt, welche nicht nur eine intensiv diskutierte Richtung in der Planungstheorie ist, sondern eben auch alltägliche Praxis. Eine Möglichkeit, sich kommunikative Aushandlungsprozesse und kooperative Zusammenarbeit in der räumlichen Planung neu zu erschließen, besteht in der Hinzunahme bisher nicht oder wenig bedachter theoretischer und methodischer Ansätze: So beschäftigen sich Meike Levin-Keitel, Bettina Lelong und Thomas Thaler mit systemischen Strukturaufstellungen zur Darstellung von Macht in der Planungspraxis. Dabei wird gezeigt, dass diese Methode sowohl einen praktischen als auch einen wissenschaftlichen Mehrwert hat, welcher beispielsweise in der Visualisierung komplexer Akteurskonstellationen und damit in der Generierung neuen Wissens besteht. Ebenfalls auf die Generierung neuen Wissens über die Praxis kooperativer Planung gerichtet ist der Beitrag von Martin Sondermann. Hier wird die kooperative Zusammenarbeit von Akteuren als Ausdruck lokaler Planungskultur neu interpretiert. Dabei wird eine auf empirischen Beobachtungen basierende Theorie über Planungskulturen als spezifische Sinnsysteme aufgestellt.

Eine kritische Reflexion der wissenschaftlichen Ergebnisse der letzten 13 Jahre wird im abschließenden Beitrag vollzogen. Christian Diller und Thomas Thaler analysieren den planungswissenschaftlichen Output seit 2003 und stellen eine Diskrepanz zwischen theoriebasierter Forschung und der Planungspraxis fest: In den Untersuchungen gelingt die empirische Überprüfung der Theorien nur teilweise. Interessant sind hierbei auch die unterschiedlichen Schwerpunkte der deutschsprachigen Zeitschriften hinsichtlich ihrer Theoriegeleitetheit, disziplinären Ausrichtung und ihrem Wandel im Laufe der Zeit.

In diesem Heft werden somit immanente Fragen zum Verhältnis unterschiedlicher Wissens- und Handlungsfelder zueinander, zum facheigenen Anspruch auf Transdisziplinarität und der Ungleichheit von Theorie und Praxis explizit verhandelt und empirisch neu ausgeleuchtet. Dabei erhebt dieses Schwerpunktheft keinen Anspruch auf Vollständigkeit, vielmehr erhoffen wir uns, die Diskussion über Planung als Disziplin im Spannungsfeld unterschiedlicher Praktiken, vielfältiger theoretischer Ansätze und raum- und planungsbezogener Wissenschaften anzuregen und die Leserinnen und Leser einzuladen, einen Moment darüber zu sinnieren, wie sich das Berufsfeld und die Disziplin der räumlichen Planung eigentlich darstellt. Wir wünschen eine anregende Lektüre.

\section{Literatur}

Alexander, E. R. (1997): A mile or a millimeter? Measuring the 'planning theory-practice gap'. In: Environment and Planning B: Planning and Design 24, 1, 3-6.

Alexander, E. R. (2010): Introduction: Does planning theory affect practice, and if so, how? In: Planning Theory 9, 2, 99-107.

Allmendinger, P. (2009): Planning theory. Basingstoke.

Allmendinger, P.; Tewdwr-Jones, M. (1997): Mind the gap: planning theory-practice and the translation of knowledge into action a comment on Alexander (1997). In: Environment and Planning B: Planning and Design 24, 6, 802-806.

Gualini, E. (2010): Planungstheorie. In: Henckel, D.; Von Kuczkowski, K.; Lau, P.; Pahl-Weber, E.; Stellmacher, F. (Hrsg.): Planen Bauen - Umwelt. Ein Handbuch. Wiesbaden, 365-369.

Healey, P. (1996): The communicative turn in planning theory and its implications for spatial strategy formations. In: Environment and Planning B: Planning and Design 23, 2, 217-234.

Innes de Neufville, J. (1983): Planning Theory and Practice: Bridging the Gap. In: Journal of Planning Education and Research 3, 1, 3545.

Levin-Keitel, M.; Sondermann, M. (2015): Räumliches Planen als kulturelles Handeln: Planungskultur als analytischer Ansatz. In: Othengrafen, F.; Sondermann, M. (Hrsg.): Städtische Planungskulturen im Spiegel von Konflikten, Protesten und Initiativen. Berlin, 31-58. = Reihe Planungsrundschau 23.

Lord, A. (2014): Towards a non-theoretical understanding of planning. In: Planning Theory 13, 1, 26-43.

Pissourios, I. A. (2013): Whither the Planning Theory-Practice Gap? A Case Study on the Relationship between Urban Indicators and 
Planning Theories. In: Theoretical and Empirical Researches in Urban Management 8, 2, 80-92.

Reuter, W. (2000): Zur Komplementarität von Diskurs und Macht in der Planung. In: disP - The Planning Review 36, 141, 4-16.
Van de Ven, A. H.; Johnson, P. E. (2006): Knowledge for Theory and Practice. In: Academy of Management Review 31, 4, 802-821. 